\title{
INVESTIGAÇÕES SOBRE PONTOS DE MÁXIMO E MÍNIMO DE FUNÇÕES LUCRO POLINOMIAIS DE $4^{\circ}$ GRAU GENÉRICA
}

\section{INVESTIGATIONS ON GENERIC POLYNOMIAL 4RD ORDER PROFIT FUNCTIONS: MAXIMUM AND MINIMUM POINTS}

\author{
Diógenes Bosquetti ${ }^{\mathrm{I}}$ \\ Alexandre de Souza Fernandes ${ }^{\text {II }}$ \\ Péricles Bosquetti ${ }^{\mathrm{III}}$ \\ Omar Maluf ${ }^{\mathrm{IV}}$ \\ Maurício Angeloni ${ }^{\mathrm{V}}$
}

\begin{abstract}
RESUMO
A função lucro é uma função importante na sustentabilidade da empresa. O lucro acontece para valores positivos de $\mathrm{L}(\mathrm{x})$ e prejuízo quando $\mathrm{L}(\mathrm{x})<0$. Os pontos de máximo e mínimo de uma função lucro descrita por uma função polinomial genérica do $4^{\circ}$ grau são determinados. Neste cenário mais realista, efeitos, comportamentos, tendências podem ser previstos. Casos particulares e de interesse também estão determinados nesse estudo, traçando um panorama e proporcionando instrumento para aplicação em casos reais e de interesse, com a variação do parâmetro sob investigação. Dentre estes casos, destacam-se as equações biquadradas e, também, os casos em que o interesse comportamental só tem significado para valores positivos do parâmetro. Nestes casos, restrições adicionais devem ser consideradas na análise.
\end{abstract}

Palavras-chave: Administração. Gestão Financeira. Matemática. Funções. Polinômios.

\begin{abstract}
The profit function is an important function in the company's sustainability. The Profit occurs for positive values of $\mathrm{L}(\mathrm{x})$ and loss when $\mathrm{L}(\mathrm{x})<0$. The Profit Function Maximum and Minimum Points' described by a generic polynomial function of the 4th degree is determined. In this realistic scenario, effects, behaviors, trends can be predicted. Particular cases are also discussed in this study, allowing to construct a richer panorama and providing an instrument for application in real cases, with the variation of the parameter under investigation " $x$ ". Among these cases, we highlight the bi-square equations and also the cases in which the behavioral interest has only meaning for positive values $(x \geq 0)$. In these cases, additional restrictions should be considered in the analysis.

\footnotetext{
I Prof. Pós-Dr. da Supervisão Regional 9: Ribeirão Preto/Franca/Barretos - São Paulo - Brasil. E-mail: diogenes.bosquetti@cps.sp.gov.br

II Prof. Me. da Faculdade de Tecnologia (FATEC) de Sertãozinho - São Paulo - Brasil. E-mail: alexandre.fernandes3@fatec.sp.gov.br

III Prof. Dr. da Faculdade de Tecnologia (FATEC) de Sertãozinho - São Paulo - Brasil. E-mail: pericles.bosquetti@fatec.sp.gov.br

IV Prof. Dr. da Faculdade de Tecnologia (FATEC) de Sertãozinho - São Paulo - Brasil. E-mail: omar.maluf@fatec.sp.gov.br

v Prof. Dr. da Faculdade de Tecnologia (FATEC) de Sertãozinho - São Paulo - Brasil. E-mail: mauricio.angeloni@fatec.sp.gov.br
} 


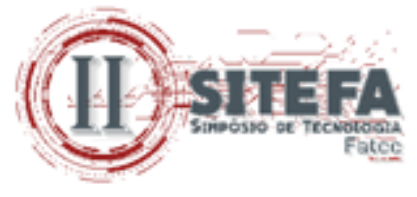

Keywords: Administration. Financial management. Mathematics. Functions. Polynomials.

Data de submissão do artigo: 29/06/2019.

Data de aprovação do artigo: 01/10/2019.

DOI:

\section{INTRODUÇÃO}

Entende-se por "Gestão Financeira de uma Empresa" (IUGU, 2017) ao conjunto de procedimentos e ações administrativas vinculados à análise, ao controle e ao planejamento de todas as atividades financeiras de uma empresa, indústria, comércio, corporações em geral. Tendo em vista a necessidade de sistematizar tais procedimentos, bem como utilizar conceitos matemáticos que possam quantizar tais ações, define-se a função lucro " $L(x)$ " (OLIVEIRA, 2016), como sendo a diferença entre duas outras funções nessa mesma variável "x":

$$
L(x)=R(x)-C(x) .
$$

A função " $R(x)$ " é denominada "função receita" enquanto " $C(x)$ " é a função custo. Assumindo que a função lucro seja adequadamente descrita por um polinômio do $4^{\circ}$ grau na variável "x", os coeficientes "a", "b", "c", "d" e "e", todos independentes entre si e de "x", temos:

$$
L(x)=a x^{4}+b x^{3}+c x^{2}+d x+e \quad \operatorname{com} a \neq 0 .
$$

Trata-se, portanto, de uma generalização das funções lucro usualmente considerada nas análises financeiras tradicionais. Tal generalização possibilita a inclusão de efeitos, comportamentos, tendências, agregação de valores que só podem ser explicados ao se considerar funções mais complexas, criando cenários mais realistas de mercado (BOSQUETTI et al, 2018). Em artigo anterior, os pontos de equilíbrio de uma função lucro do $4^{\circ}$ grau foram investigados (BOSQUETTI et al, 2019).

Neste trabalho, os pontos locais de máximo e mínimo para a função genérica do $4^{\circ}$ grau são determinados através a aplicação da metodologia usual para a obtenção dos mesmos (SODRÉ, 2006a; 2006b) Admitindo-se que o parâmetro $a \neq 0$, as derivadas de primeira, segunda, terceira e quarta ordem da função lucro na variável " $x$ ", são dadas como se seguem:

$$
\begin{gathered}
L^{\prime}(x)=d L(x) / d x=4 a x^{3}+3 b x^{2}+2 c x+d, \\
L^{\prime \prime}(x)=d^{2} L(x) / d x^{2}=12 a x^{2}+6 b x+2 c, \\
L^{\prime \prime \prime}(x)=d^{3} L(x) / d x^{3}=24 a x+6 b, \\
L^{\prime \prime \prime \prime}(x)=d^{4} L(x) / d x^{4}=24 a .
\end{gathered}
$$

As derivadas de ordem igual ou superior a cinco anulam a função lucro “ $L(x)$ ”. Tendo em vista que a derivada de primeira ordem $L^{\prime}(x)=0$ nos pontos de mínimo ou máximo relativo, existe a necessidade de se aplicar o teste da derivada de segunda ordem. Seja $x=v$, o valor da variável " $x$ " tal que $L^{\prime}(v)=0$ e $L^{\prime \prime}(v)>0$. Neste caso, a função $L(x)$ tem um ponto de mínimo local neste ponto. Se em $x=v, L^{\prime}(v)=0$ e $L^{\prime \prime}(v)<0$, a função lucro $L(x)$ tem um ponto de máximo local neste ponto. Quando $L^{\prime}(v)=L^{\prime \prime}(v)=0$, o teste é inclusivo, 


\section{(iil) $\sin \frac{\pi}{1}$}

devendo considerar-se a derivada de ordem seguinte para nova análise. Neste último caso, se $L^{\prime}(v)=L^{\prime \prime}(v)=0$ e $L^{\prime \prime \prime}(v) \neq 0$, o ponto é de inflexão. Se $L^{\prime}(v)=L^{\prime \prime}(v)=L^{\prime \prime \prime}(v)=0$ é necessário considerar a derivada de quarta ordem em relação a variável "x" para determinar se o ponto é de máximo ou mínimo. Neste caso, $L^{\prime \prime \prime \prime}(v)=24 a$, de modo que $x=v$, será ponto de máximo se $a<0$ e ponto de mínimo se $a>0$.

\section{PONTOS CRÍTICOS DE UMA FUNÇÃO LUCRO DE $4^{\circ}$ GRAU}

Tendo em vista o objeto do estudo aqui realizado, os pontos críticos da função lucro acontecem toda vez que sua derivada de primeiro grau em relação a " $x$ " se anula, ou seja, $L^{\prime}(x)=0$. Neste cenário, tais pontos são as raízes da equação de terceiro grau:

$$
4 a x^{3}+3 b x^{2}+2 c x+d=0 .
$$

Uma vez que o parâmetro $a \neq 0$, podemos dividir toda a equação pelo coeficiente que multiplica a terceira potência em " $x "$, ou seja, o termo " $4 a$ ". Assim procedendo, a equação anterior pode ser reescrita como:

$$
x^{3}+\frac{3 b}{4 a} x^{2}+\frac{c}{2 a} x+\frac{d}{4 a}=0 .
$$

Realizando a seguinte mudança de variável:

$$
z=x+\frac{b}{4 a}
$$

o termo quadrático da equação (8) se anula, obtendo a denominada forma reduzida da equação de terceiro grau (ANDREATINI, 2019):

$$
z^{3}+p z+q=0
$$

onde as quantidades "p" e "q" são definidas em termos dos parâmetros “a", "b", "c" e "d" como se segue:

$$
\begin{gathered}
p=\frac{c}{2 a}-\frac{3 b^{2}}{16 a^{2}}, \\
q=\frac{d}{4 a}-\frac{b c}{8 a^{2}}+\frac{b^{3}}{32 a^{3}} .
\end{gathered}
$$
definido por:

De posse destas duas últimas quantidades, é possível a construção do discriminante " $\Delta$ ",

$$
\Delta=\frac{q^{2}}{4}+\frac{p^{3}}{27}
$$

Analisando o sinal deste discriminante, temos que quando $\Delta>0$, a equação de terceiro grau apresenta uma solução real e duas complexas conjugadas entre si. Quando $\Delta<0$, a referida equação possui três raízes reais (CYBERINI, 2018). Finalmente, se $\Delta=0$, duas situações podem ocorrer: Se as quantidades $p=q=0$, temos três raízes reais e iguais $x_{1}=x_{2}=x_{3}=$ $b / 4 a$. Se $q^{2} / 4=-p^{3} / 27 \neq 0$, temos duas soluções reais (raiz dupla), a saber: 


$$
\begin{gathered}
x_{1}=-\frac{b}{4 a}-2 \sqrt[3]{\frac{q}{2}} \\
x_{2}=x_{3}=-\frac{b}{4 a}+\sqrt[3]{\frac{q}{2}} .
\end{gathered}
$$

A seguir apresentamos as soluções para os valores positivos e negativos para o discriminante " $\Delta$ ".

\subsection{Pontos Críticos de uma Função Lucro de $4^{\circ}$ Grau para a Condição $\Delta>0$}

Na condição onde o discriminante $\Delta=q^{2} / 4+p^{3} / 27>0$, a equação terá uma raiz real " $x_{1}$ " e duas outras complexas conjugadas " $x_{2}$ " $\mathrm{e}$ " $x_{3}$ ", a seguir apresentadas:

$$
\begin{gathered}
x_{1}=-\frac{b}{4 a}+\sqrt[3]{-\frac{q}{2}+\sqrt{\Delta}}-\sqrt[3]{\frac{q}{2}+\sqrt{\Delta}} \\
x_{2}=-\frac{b}{4 a}-\frac{1}{2}(1-i \sqrt{3}) \sqrt[3]{-\frac{q}{2}+\sqrt{\Delta}}+\frac{1}{2}(1+i \sqrt{3}) \sqrt[3]{\frac{q}{2}+\sqrt{\Delta}} \\
x_{3}=-\frac{b}{4 a}-\frac{1}{2}(1+i \sqrt{3}) \sqrt[3]{-\frac{q}{2}+\sqrt{\Delta}}+\frac{1}{2}(1-i \sqrt{3})_{3}^{\sqrt[3]{\frac{q}{2}+\sqrt{\Delta}}} .
\end{gathered}
$$

Salvo exceção de casos excepcionais, as grandezas associadas às funções lucros são reais, de forma que as raízes complexas não encontram significado na análise financeira em questão. Para determinar se " $x_{1}$ " é ponto de máximo ou mínimo, é necessário substituir a equação (16) na expressão para a derivada de segunda ordem na variável " $x$ " para a função lucro, ou seja, em $L^{\prime \prime}(x)=12 a x^{2}+6 b x+2 c$. Assim procedendo, temos:

$$
L^{\prime \prime}\left(x_{1}\right)=12 a\left(-\frac{b}{4 a}+\varepsilon-\eta\right)^{2}+6 b\left(-\frac{b}{4 a}+\varepsilon-\eta\right)+2 c,
$$

onde as quantidades " $\varepsilon$ " $\mathrm{e}$ "” são definidas respectivamente como:

$$
\begin{gathered}
\varepsilon=\sqrt[3]{-\frac{q}{2}+\sqrt{\Delta}}, \\
\eta=\sqrt[3]{\frac{q}{2}+\sqrt{\Delta}} .
\end{gathered}
$$

Desenvolvendo algebricamente a equação (19), encontramos que

$$
L^{\prime \prime}\left(x_{1}\right)=12 a\left(\varepsilon^{2}+\eta^{2}\right)+\frac{3 b^{2}}{4 a}-2 c .
$$

Se $L^{\prime \prime}\left(x_{1}\right)>0$, então " $x_{1}$ " é ponto de mínimo. Se $L^{\prime \prime}\left(x_{1}\right)<0$, então " $x_{1}$ " é ponto de máximo. Se $L^{\prime \prime}\left(x_{1}\right)=0$, devemos investigar o valor da derivada terceira da função lucro no ponto " $x_{1}$ ". Assim procedendo, a expressão matemática para $L^{\prime \prime}(x)=24 a x+6 b$ assume a forma:

$$
L^{\prime \prime \prime}\left(x_{1}\right)=24 a(\varepsilon-\eta)
$$




\section{(iil) $\sin =\frac{1}{1 \cdot}$}

Se $L^{\prime \prime \prime}\left(x_{1}\right) \neq 0$, ou seja, se $\varepsilon \neq \eta$, então " $x_{1}$ " é ponto de inflexão. Se $L^{\prime \prime \prime}\left(x_{1}\right)=0$, a análise deve prosseguir para a derivada de quarta ordem na variável " $x$ ", a qual resulta em $L^{\prime \prime \prime \prime}(x)=24 a$, a qual determinará que " $x_{1}$ " será ponto de máximo se $a<0$ e ponto de mínimo se $a>0$.

\subsection{Forma trigonométrica dos pontos críticos de uma função lucro de $4^{\circ}$ grau para a condição $\Delta<\mathbf{0}$}

A forma trigonométrica dos pontos críticos de uma função de $4^{\circ}$ grau genérica pode ser encontrada aplicando-se a identidade dada pela fórmula de Euler (UFGRS-IME, 2019):

$$
e^{ \pm i \pi / 3}=\cos (\pi / 3) \pm i \cdot \operatorname{sen}(\pi / 3)=\frac{1}{2}(1 \pm i \sqrt{3})
$$

e realizando uma mudança de variável associada aos termos existentes dentro da raiz cúbica, temos:

$$
\frac{q}{2} \pm \sqrt{\Delta}=\rho[\cos (\varphi) \pm i \cdot \sin (\varphi)]=\rho e^{ \pm i \varphi} .
$$

A relação entre as grandezas " $\rho$ " e " $\varphi$ " em função de grandezas já conhecidas, particularmente "p" e "q" definidos pelas equações (11) e (12), podem ser encontradas através da resolução do seguinte sistema de equações:

$$
\left\{\begin{array}{l}
\frac{q}{2}+\sqrt{\Delta}=\rho e^{i \varphi} \\
\frac{q}{2}-\sqrt{\Delta}=\rho e^{-i \varphi}
\end{array} .\right.
$$

Somando as duas equações do sistema anterior, obtemos $q=2 \rho \cos (\varphi)$. De forma análoga, através da subtração de ambas as equações, obtemos que o discriminante $\sqrt{\Delta}=$ i $2 \rho \operatorname{sen}(\varphi)$. Continuando a análise, temos, pela segunda equação do sistema, que

$$
e^{i \varphi}=\rho /[q / 2-\sqrt{\Delta}] .
$$

Substituindo esta relação na primeira equação do sistema de equações, encontramos uma expressão para a grandeza " $\rho$ ", em termos de "p" e "q", a saber:

$$
\rho^{2}=\frac{q^{2}}{4}-\Delta=\frac{p^{3}}{27}
$$

Realizando a divisão entre as equações do sistema de equações apresentado, obtemos os seguintes resultados:

$$
\begin{gathered}
e^{i 2 \varphi}=\frac{q / 2+\sqrt{\Delta}}{q / 2-\sqrt{\Delta}}, \\
e^{-i 2 \varphi}=\frac{q / 2-\sqrt{\Delta}}{q / 2+\sqrt{\Delta}} .
\end{gathered}
$$

Desta forma, da soma das equações (29) e (30) uma expressão para o $\cos (2 \varphi)$, a saber: 


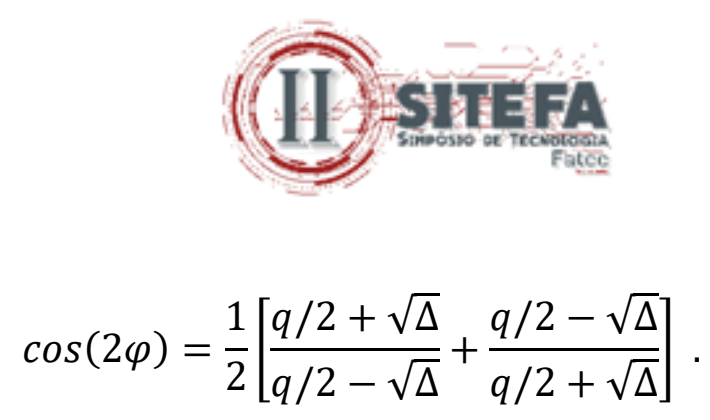

Realizando algumas manipulações algébricas, e utilizando-se o conceito de função trigonométrica inversa (UNICAMP-IME, 2018), verifica-se que:

$$
\varphi=\frac{1}{2} \arccos \left[\frac{q^{2} / 4+\Delta}{q^{2} / 4-\Delta}\right]=\frac{1}{2} \arccos \left[1+\frac{27 q^{2}}{2 p^{3}}\right] .
$$

As raízes da equação $L^{\prime}(x)=0$, determinada pelas equações (16), (17) e (18) podem ser reescritas, nestas novas variáveis, como:

$$
\begin{gathered}
x_{1}=-\frac{b}{4 a}-\rho^{1 / 3} e^{-i \varphi / 3}-\rho^{1 / 3} e^{i \varphi / 3} \\
x_{2}=-\frac{b}{4 a}+e^{-i \pi / 3} \rho^{1 / 3} e^{-i \varphi / 3}+e^{i \pi / 3} \rho^{1 / 3} e^{i \varphi / 3} \\
x_{3}=-\frac{b}{4 a}+e^{i \pi / 3} \rho^{1 / 3} e^{-i \varphi / 3}+e^{-i \pi / 3} \rho^{1 / 3} e^{i \varphi / 3} .
\end{gathered}
$$

Nas equações anteriores, a grandeza " $\rho^{1 / 3}$ " aparece como fator comum nos dois últimos termos das equações, podendo ser colocado em evidência. Nas duas últimas equações, é possível agrupar o produto das exponenciais em uma única, pois ambas apresentam a mesma base. Somando-se as potências de cada uma, temos:

$$
\begin{gathered}
x_{1}=-\frac{b}{4 a}-\rho^{1 / 3}\left(e^{-i \varphi / 3}+e^{i \varphi / 3}\right), \\
x_{2}=-\frac{b}{4 a}+\rho^{1 / 3}\left(e^{-i(\pi / 3+\varphi / 3)}+e^{i(\pi / 3+\varphi / 3)}\right), \\
x_{3}=-\frac{b}{4 a}+\rho^{1 / 3}\left(e^{-i(\pi / 3-\varphi / 3)}+e^{i(\pi / 3-\varphi / 3)}\right) .
\end{gathered}
$$

Para uma quantidade genérica " $\theta$ ", é válida a identidade $e^{i \theta}+e^{-i \theta}=2 \cdot \cos (\theta)$. Desta forma, as expressões anteriores assumem a forma final:

$$
\begin{gathered}
x_{1}=-\frac{b}{4 a}-2 \rho^{1 / 3} \cos (\varphi / 3), \\
x_{2}=-\frac{b}{4 a}+2 \rho^{1 / 3} \cos (\pi / 3+\varphi / 3), \\
x_{3}=-\frac{b}{4 a}+2 \rho^{1 / 3} \cos (\pi / 3-\varphi / 3) .
\end{gathered}
$$

Estas últimas equações são a forma trigonométrica das raízes “ $x_{1}$ ", “ $x_{2}$ " e " $x_{3}$ ". 


\section{(110)}

\subsection{Pontos críticos de uma função lucro de $4^{\circ}$ grau para a condição $\Delta<0$}

Na condição onde o discriminante $\Delta=q^{2} / 4+p^{3} / 27<0$, as raízes " $x_{1}$ ", " $x_{2}$ " e " $x_{3}$ " são reais, necessitando serem todas consideradas nas análises de gestão financeira da empresa no estudo sobre lucros máximo e mínimo.

\subsubsection{Análise da função lucro de $4^{\circ}$ grau para a raiz " $x_{1}$ "}

Na investigação sobre os pontos críticos, substitui-se a formulação matemática da raiz “ $x_{1}$ ”, obtida na seção anterior, na expressão $L^{\prime \prime}(x)=12 a x^{2}+6 b x+2 c$. Assim procedendo, temos:

$$
L^{\prime \prime}\left(x_{1}\right)=12 a\left(-\frac{b}{4 a}-2 \rho^{1 / 3} \cos \left(\frac{\varphi}{3}\right)\right)^{2}+6 b\left(-\frac{b}{4 a}-2 \rho^{1 / 3} \cos \left(\frac{\varphi}{3}\right)\right)+2 c .
$$

Realizando o desenvolvimento algébrico desta expressão, encontramos:

$$
L^{\prime \prime}\left(x_{1}\right)=48 a \rho^{2 / 3} \cos ^{2}\left(\frac{\varphi}{3}\right)-\frac{3 b^{2}}{4 a}+2 c .
$$

Pelo teste da derivada segunda, temos que se $L^{\prime \prime}\left(x_{1}\right)>0$, então " $x_{1}$ " é ponto de mínimo; se $L^{\prime \prime}\left(x_{1}\right)<0$, então " $x_{1}$ " é ponto de máximo; se $L^{\prime \prime}\left(x_{1}\right)=0$, devemos derivar novamente a função lucro, obtendo, neste caso, a expressão $L^{\prime \prime \prime}(x)=24 a x+6 b$, estudando o valor de $L^{\prime \prime \prime}(x)$ no ponto " $x_{1}$ ". Assim procedendo, verifica-se que:

$$
L^{\prime \prime \prime}\left(x_{1}\right)=-48 a \rho^{1 / 3} \cos \left(\frac{\varphi}{3}\right) \text {. }
$$

O ponto " $x_{1}$ " é ponto de inflexão se $L^{\prime \prime}$ " $\left(x_{1}\right) \neq 0$. Caso $L^{\prime \prime}$ " $\left(x_{1}\right)=0$, a análise prossegue para a ordem seguinte, $L^{\prime \prime \prime \prime}(x)=24 a$, a qual determinará que " $x_{1}$ ” será ponto de máximo quando $a<0$ e ponto de mínimo quando $a>0$.

\subsubsection{Análise da função lucro de $4^{\circ}$ Grau para a raiz " $x_{2}$ "}

Substituindo a forma trigonométrica da raiz " $x_{2}$ ", obtida na seção anterior, na expressão matemática da derivada de segunda ordem da função lucro $L^{\prime \prime}(x)$, obtemos:

$$
L^{\prime \prime}\left(x_{2}\right)=12 a\left(2 \rho^{1 / 3} \cos \left(\frac{\pi}{3}+\frac{\varphi}{3}\right)-\frac{b}{4 a}\right)^{2}+6 b\left(2 \rho^{1 / 3} \cos \left(\frac{\pi}{3}+\frac{\varphi}{3}\right)-\frac{b}{4 a}\right)+2 c .
$$

Realizando o desenvolvimento algébrico desta expressão, encontramos:

$$
L^{\prime \prime}\left(x_{2}\right)=48 a \rho^{2 / 3} \cos ^{2}\left(\frac{\pi}{3}+\frac{\varphi}{3}\right)-\frac{3 b^{2}}{4 a}+2 c .
$$

Aplicando o teste da derivada segunda para a função lucro, temos que se $L^{\prime \prime}\left(x_{2}\right)<0$, então " $x_{2}$ " é ponto de máximo; se $L^{\prime \prime}\left(x_{2}\right)>0$, então " $x_{2}$ " é ponto de mínimo; se $L^{\prime \prime}\left(x_{2}\right)=0$, nada se pode afirmar. Considerando a derivada de terceira ordem na variável " $x$ " para a função lucro, $L^{\prime \prime}(x)$, temos, no ponto " $x=x_{2}$ ", que: 


$$
\begin{aligned}
& \text { (11) SHIEFA } \\
& L^{\prime \prime \prime}\left(x_{2}\right)=48 a \rho^{1 / 3} \cos \left(\frac{\pi}{3}+\frac{\varphi}{3}\right) \text {. }
\end{aligned}
$$

O ponto " $x_{2}$ " é ponto de inflexão se $L^{\prime \prime \prime}\left(x_{2}\right) \neq 0$. Caso $L^{\prime \prime}\left(x_{2}\right)=0$, analisa-se o sinal de $L^{\prime \prime \prime \prime}(x)=24 a$. Neste cenário, " $x_{2}$ ” será ponto de mínimo quando $a>0$ e ponto de máximo quando $a<0$.

\subsubsection{Análise da função lucro de $4^{0}$ grau para a raiz " $x_{3}$ "}

A dinâmica associada à raiz " $x_{3}$ " é idêntica à já apresentada para " $x_{2}$ ". Desta forma, a derivada de segunda e de terceira ordem da função lucro apresenta-se na forma:

$$
\begin{gathered}
L^{\prime \prime}\left(x_{3}\right)=48 a \rho^{2 / 3} \cos ^{2}\left(\frac{\pi}{3}-\frac{\varphi}{3}\right)-\frac{3 b^{2}}{4 a}+2 c . \\
L^{\prime \prime \prime}\left(x_{3}\right)=48 a \rho^{1 / 3} \cos \left(\frac{\pi}{3}-\frac{\varphi}{3}\right) .
\end{gathered}
$$

As considerações para esta raiz são idênticas às aplicadas a " $x_{2}$ " não sendo necessário sua replicação.

\section{FUNÇÕES LUCRO DESCRITOS POR POLINÔMIOS BIQUADRADOS}

Um caso particular, mas de grande interesse na literatura trata-se da situação onde os coeficientes $b=d=0$, os quais multiplicam respectivamente as potências " $\mathrm{x}$ " $\mathrm{e}$ " $\mathrm{x}$ " da função lucro se anulam. Neste caso, a função lucro é descrita matematicamente por um polinômio biquadrado (SO MATEMATICA, 2019):

$$
L(x)=a x^{4}+c x^{2}+e \quad \operatorname{com} a \neq 0 .
$$

As derivadas de primeira, segunda, terceira e quarta ordem da função lucro na variável "x", são dadas como se seguem:

$$
\begin{gathered}
L^{\prime}(x)=d L(x) / d x=4 a x^{3}+2 c x, \\
L^{\prime \prime}(x)=d^{2} L(x) / d x^{2}=12 a x^{2}+2 c, \\
L^{\prime \prime \prime}(x)=d^{3} L(x) / d x^{3}=24 a x, \\
L^{\prime \prime \prime \prime}(x)=d^{4} L(x) / d x^{4}=24 a .
\end{gathered}
$$

Neste caso particular, as quantidades "p", "q" e o discriminante " $\Delta$ " as quais são definidas nas equações (11), (12) e (13) em termos dos parâmetros "a", "b", "c" e "d" se simplificam da seguinte maneira:

$$
\begin{gathered}
p=\frac{c}{2 a}, \\
q=0,
\end{gathered}
$$






De posse destas simplificações, as raízes " $x_{1}$ ", " $x_{2}$ " e " $x_{3}$ ", da função $L^{\prime}(x)=4 a x^{3}+$ $2 c x$ apresentadas nas equações (16), (17) e (18) se simplificam, podendo ser expressas por:

$$
\begin{gathered}
x_{1}=0 \\
x_{2}=-\frac{1}{2}(1-i \sqrt{3}) \sqrt{\frac{p}{3}}+\frac{1}{2}(1+i \sqrt{3}) \sqrt{\frac{p}{3}}, \\
x_{3}=-\frac{1}{2}(1+i \sqrt{3}) \sqrt{\frac{p}{3}}+\frac{1}{2}(1-i \sqrt{3}) \sqrt{\frac{p}{3}} .
\end{gathered}
$$

Realizando nova simplificação algébrica para as expressões de " $x_{2}$ " e " $x_{3}$ ”, obtemos

$$
x_{2}=-x_{3}=i \sqrt{p}=i \sqrt{\frac{c}{2 a}} .
$$

Aplicando o teste da derivada segunda para a função lucro, onde $L^{\prime \prime}(x)=12 a x^{2}+2 c$, e lembrando que $x_{1}=0$ e $x_{2}^{2}=x_{3}^{2}=-c / 2 a$, temos:

$$
\begin{gathered}
L^{\prime \prime}\left(x_{1}\right)=2 c, \\
L^{\prime \prime}\left(x_{2}\right)=L^{\prime \prime}\left(x_{3}\right)=-4 c .
\end{gathered}
$$

Deste modo, temos que se a derivada segunda for negativa, então o ponto crítico será de máximo. Por outro lado, se a derivada segunda $L^{\prime \prime}(x)>0$, então o ponto estudado é de mínimo. Neste caso particular, quando $c>0$, o ponto " $x_{1}$ " é de mínimo enquanto " $x_{2}$ " e " $x_{3}$ " são de máximo. Quando $c<0$, a situação descrita na frase anterior se inverte. Na condição $c=0$, nada se pode afirmar. Tendo em vista que esta última opção resulta em três raízes reais e nulas, ou seja, para $c=0, x_{1}=x_{2}=x_{3}=0$ (raiz tripla) e consequentemente $L^{\prime \prime \prime}\left(x_{1}\right)=L^{\prime \prime \prime}\left(x_{2}\right)=$ $L^{\prime \prime \prime}\left(x_{3}\right)=24 a x=0$, é necessário estudar-se o sinal da derivada de quarta ordem para a função lucro. Neste caso, $L^{\prime \prime \prime \prime}(x)=24 a$ e as raízes serão ponto de mínimo quando $a>0$ e ponto de máximo quando $a<0$.

\section{RESTRIÇõES ADICIONAIS À EXISTÊNCIA DE PONTOS DE CRÍTICOS PARA FUNÇÕES LUCRO DE GRANDEZAS ESTRITAMENTE POSITIVAS}

Uma situação particular, mas de grande interesse na gestão financeira acontece quando os valores da grandeza " $x$ ” devem ser estritamente positivos. Neste caso em particular, algumas restrições adicionais devem ser consideradas para a efetiva existência dos pontos de lucro máximo ou mínimo. Isso, pois, não deverão ser considerados pontos críticos que ocorrem para valores de $x<0$.

Para as restrições adicionais de existência dos pontos críticos possam ser encontradas, devemos considerar as raízes da equação $L^{\prime}(x)=0$, a qual resulta em três possíveis raízes " $x_{1}$ ", " $x_{2}$ " e " $x_{3}$ ". No caso em que o discriminante $\Delta=q^{2} / 4+p^{3} / 27>0$, a equação terá apenas uma raiz real " $x_{1}$ ", a qual terá que ser necessariamente maior ou igual a zero. Desta forma, a seguinte restrição adicional é conseguida: 


$$
\begin{aligned}
& \text { (110) } \\
& \sqrt[3]{-\frac{q}{2}+\sqrt{\Delta}}-\sqrt[3]{\frac{q}{2}+\sqrt{\Delta}} \geq \frac{b}{4 a} .
\end{aligned}
$$

No caso em que $\Delta=0$ e as quantidades $p=q=0$, temos três raízes reais e iguais e a condição adicional para a existência de pontos críticos será:

$$
b / a \geq 0 \text {. }
$$

Se $\Delta=0$ mas $q^{2} / 4=-p^{3} / 27 \neq 0$, a existência dos pontos críticos acontecerá se e somente se forem satisfeitas ao menos uma das duas condições:

$$
\sqrt[3]{q} \leq-\frac{\sqrt[3]{2} b}{8 a}, \quad \quad \text { e/ou } \quad \sqrt[3]{q} \geq \frac{\sqrt[3]{2} b}{4 a}
$$

Na condição $\Delta<0$, as raízes " $x_{1}$ ", " $x_{2}$ " e " $x_{3}$ " são reais e a existência de algum ponto crítico acontecerá se for satisfeita pelo menos uma das seguintes condições:

$$
\rho^{1 / 3} \cos (\varphi / 3) \leq-\frac{b}{8 a}, \quad \text { e/ou } \quad \rho^{1 / 3} \cos (\pi / 3 \pm \varphi / 3) \geq \frac{b}{8 a}
$$

A satisfação de mais de uma condição implica na existência de mais de um ponto crítico. Evidentemente as análises já realizas nas seções anteriores para a determinação de ponto de máximo, mínimo ou de inflexão continuam válidas e aplicáveis neste caso.

\section{DISCUSSÃO E CONCLUSÃO}

Uma função lucro polinomial do $4^{\circ}$ grau foi estudada no intuito de se estabelecer seus pontos críticos para os casos em que o discriminante $\Delta=q^{2} / 4+p^{3} / 27$ assume valores positivos, negativos ou nulos. Estes cenários permitem considerar efeitos, comportamentos, propriedades não explicáveis para funções lucro polinomiais de ordens menores, proporcionando um cenário mais realístico da realidade. Igualmente deve ser ressaltado que este estudo geral tanto engloba quanto generaliza estudos de lucro máximo ou mínimo usualmente desenvolvidos na literatura, bem como investigados anteriormente por (BOSQUETTI et al., 2018) para uma função lucro dada por um polinômio do $3^{\circ}$ grau. Igualmente este trabalho complementa o artigo sobre pontos de equilíbrio de uma função lucro de $4^{\circ}$ grau (BOSQUETTI et al, 2019).

De uma forma geral, as funções de $4^{\circ}$ grau apresentam um ponto crítico quando $\Delta>0$, um ou dois pontos críticos para $\Delta=0$ e três pontos críticos se o discriminante $\Delta<0$. Neste último caso, aplica-se a formulação trigonométrica para as soluções encontradas para $L^{\prime}(x)=$ $d L(x) / d x=4 a x^{3}+3 b x^{2}+2 c x+d$. Para a determinação da natureza deste ponto crítico (se ele é ponto de máximo, mínimo ou de inflexão), aplica-se procedimentos investigativos associados ao teste de derivada n-ésima. É fato, porém, que a combinação dos valores dos parâmetros "a", "b", "c" e "d" determinação o caso em questão.

Neste trabalho são considerados alguns casos particulares e de interesse na literatura: Funções Lucro descritos por funções polinomiais biquadradas e, também, casos onde o parâmetro investigado assume valores estritamente maiores ou iguais a zero. Em ambos os casos, algumas simplificações e/ou condições restritivas adicionais são obtidas, adequando-os 


\section{(1010)}

a tais particularidades. Evidentemente, o parâmetro " $x$ " referenciado ao longo de todo o trabalho apresenta significado real variável caso a caso.

\section{REFERÊNCIAS}

ANDREATINI, A. A solução das equações de $3^{\circ}$ e $4^{\mathbf{0}}$ grau Cardano e Tartaglia. Última Atualização 2019. Disponível em: http://www.andreatini.com.br/cardano.htm. Acesso em 22 jun 2019.

BOSQUETTI, D. et al. Investigações sobre Funções Lucro de $3^{\circ}$ Grau Genérica: pontos de equilíbrio e de lucros máximo e mínimo. 2018. Disponível em: http://sitefa.fatecsertãozi nho.edu.br/index.php/sitefa/article/view/17/27. Acesso em: 21 jun. 2019.

Investigações sobre pontos de equilíbrio de funções lucro polinomiais de $4^{\circ}$ grau genérica. 2019. Artigo Submetido ao II Simpósio de Tecnologia da Faculdade de Tecnologia de Sertãozinho (II SITEFA). Disponível em: https://www.fatecsertaozinho. edu.br/evento/iisimposio-de-tecnologia-da-fatec-de-sertaozinho. Acesso em: 27 jun 2019.

CYBERINI, F. H. Algoritmo da equação do quarto grau. 2018. Disponível em: https://www.blogcyberini.com/2018/06/algoritmo-equacao-quarto-grau.html. Acesso em: 5 mai 2019.

IUGU. Tudo o que você precisa saber para a gestão financeira da sua empresa. 2017. Disponível em: https://iugu.com/blog/tudo-gestao-financeira-sua-empresa/. Acesso em: 13 jun. 2019.

OLIVEIRA, M. Função receita, função lucro e ponto de equilíbrio. Contabilidade e Matemática para Negócios e Concursos. 2016. Disponível em: https://www.manoeloliveira. net.br /2016/10/funcao-receita-lucro-e-ponto-deequilibrio.html. Acesso em: 13 jun. 2019.

SODRÉ, U. Ensino Superior: cálculo: máximos e mínimos: teste da primeira derivada. 2006a. Disponível em: http://www.uel.br/projetos/matessencial/superior/calculo/maxmin/ mm02.htm. Acesso em: 18 jun. 2019.

Ensino Superior: cálculo: máximos e mínimos: teste da segunda derivada. 2006b. Disponível em: http://www.uel.br/projetos/matessencial/superior/calculo/maxmin/mm03.htm. Acesso em: 18 jun. 2019.

SO MATEMATICA. Equações biquadradas. 2019. Disponível em: https://www.somatemat ica.com.br/fundam/equacoes2/equacoes2_12.php. Acesso em: 2 mai. 2019.

UFRGS-IME. Números complexos e fórmula de Euler. 2019. Disponível em: https://www.ufrgs.br/reamat/TransformadasIntegrais/livro-af/rdnceftnx00fameros_complexos_e_fx00f3rmula_de_euler.html. Acesso em: 26 jun. 2019.

UNICAMP-IME. Funções trigonométricas inversas. 2018. Disponível em: https://www.ime.unicamp.br/ js/trighip.pdf. Acesso em: 26 jun. 2019. 